\title{
Comparison of chemical freeze-out criteria in heavy-ion collisions
}

\author{
J. Cleymans \\ UCT-CERN Research Centre and Department of Physics, University of Cape Town, Rondebosch 7701, South Africa
}

\author{
H. Oeschler \\ Darmstadt University of Technology, D-64289 Darmstadt, Germany and UCT-CERN Research Centre and Department of Physics, \\ University of Cape Town, Rondebosch 7701, South Africa \\ K. Redlich \\ Institute of Theoretical Physics, University of Wrocław, Pl. Maksa Borna 9, 50-204 Wrocław, Poland and CERN TH, \\ CH 1211 Geneva 23, Switzerland \\ S. Wheaton \\ UCT-CERN Research Centre and Department of Physics, University of Cape Town, Rondebosch 7701, South Africa and Darmstadt \\ University of Technology, D-64289 Darmstadt, Germany \\ (Received 18 November 2005; published 13 March 2006)
}

\begin{abstract}
One of the most remarkable results to emerge from heavy-ion collisions over the past two decades is the striking regularity shown by particle yields at all energies. This has led to several very successful proposals describing particle yields over a very wide range of beam energies, reaching from $1 \mathrm{~A} \mathrm{GeV}$ up to $200 \mathrm{~A} \mathrm{GeV}$, using only one or two parameters. A systematic comparison of these proposals is presented here. The conditions of fixed energy per particle, baryon+anti-baryon density, normalized entropy density as well as percolation model are investigated. The results are compared with the most recent chemical freeze-out parameters obtained in the thermal-statistical analysis of particle yields. The sensitivity and dependence of the results on parameters is analyzed and discussed. It is shown that in the energy range above the top energy of the BNL Alternating Gradient Synchrotron within present accuracies, all chemical freeze-out criteria give a fairly good description of the particle yields. However, the low energy heavy-ion data favor the constant energy per particle as a unified condition of chemical particle freeze-out. This condition also shows the weakest sensitivity on model assumptions and parameters.
\end{abstract}

DOI: 10.1103/PhysRevC.73.034905

PACS number(s): 24.10.Pa, 25.75.Dw, 12.38.Mh

\section{INTRODUCTION}

One of the most remarkable results to emerge from relativistic heavy-ion collisions over the past years is the striking regularity shown by particle yields at all beam energies. From the lowest at the GSI Schwerionen synchrotron (SIS) up to the highest energies at the BNL Relativistic Heavy Ion Collider (RHIC), all results on particle multiplicities are consistent with the assumption of chemical equilibrium in the final-state fireball produced after heavy-ion impact [1]. The particle yields are found to be described, with remarkable precision, by a thermal-statistical model that assumes approximate chemical equilibrium [1-14,17]. For a given collision energy, the thermal-statistical model with only two parameters, the temperature $(T)$ and baryon chemical potential $\left(\mu_{B}\right)$, provides a very systematic description of particle yields. As the model considers integrated particle multiplicities, it is not sensitive to local inhomogeneities and/or fluctuations as these will mainly disappear after integration over particle moneta. Local inhomogeneities have been condidered in recent years in detail in Refs. [15,16].

The partition function used in heavy-ion collisions has also been shown to be consistent with results obtained from lattice gauge theory (LGT) in the hadronic phase [18,19]. This, together with the phenomenological success of the thermalstatistical model, indicates that the resonance gas description of the hadronic phase is a remarkably accurate approximation of QCD thermodynamics in the hadronic confined phase.

Using this description as a starting point, a further interesting systematic behavior of thermal parameters has emerged from particle yields in heavy-ion collisions from SIS up to RHIC. With increasing collision energy, there is an increase of the chemical freeze-out temperature, $T$, and a corresponding decrease of the baryon chemical potential, $\mu_{B}$. In the $\left(T, \mu_{B}\right)$ plane the freeze-out parameters lie on a curve connecting the lowest data points taken at the SIS through the data points taken at the BNL Alternating Gradient Synchrotron (AGS), at the CERN Super Proton Synchrotron (SPS), on at RHIC with a temperature at $\mu_{B}=0$ that corresponds to the critical temperature expected for deconfinement in LGT [1,20,21]. Such a regular behavior of the freeze-out parameters has called for an interpretation based on the interaction dynamics. There are properties of the thermal fireball at chemical freeze-out that are common to all collision energies. Such common properties provide unified chemical freeze-out conditions in heavy-ion collisions at all energies.

The knowledge of such unified freeze-out properties is useful since:

(i) they make it possible to determine particle excitation functions and to study their systematic properties [22]. Consequently, general conclusions can be drawn about 
the energy dependence of particle production in heavyion collisions;

(ii) the freeze-out conditions are relevant for a successful description of particle spectra;

(iii) on the theoretical level the unified freeze-out conditions are relevant for the understanding of particle production dynamics. The chemical freeze-out line has been interpreted as the inelasticity line in heavy-ion collisions [23], as it separates the stage where the evolution dynamics of the collision fireball is dominated by inelastic instead of elastic processes.

The first unified chemical freeze-out criterion was recognized [24,25] when comparing the thermal parameters at SIS energy with those obtained at SPS. It was shown that the average energy per particle at SIS energy reaches approximately the same value of $1 \mathrm{GeV}$ as calculated at the critical temperature expected for deconfinement at $\mu_{B}=0$. In addition, known results for chemical freeze-out parameters at the AGS also reproduced the same value of energy per particle. Thus, it was suggested that the condition of a fixed energy per hadron is the chemical freeze-out criterion in heavy-ion collisions. More precise determinations prefer a value $\langle E\rangle /\langle N\rangle \approx 1.08 \mathrm{GeV}$.

In addition to the fixed $\langle E\rangle /\langle N\rangle$ criterion, alternative proposals have been made to describe chemical freeze-out in heavy-ion collisions at all energies [26-32]:

(i) a fixed value for the sum of baryon and antibaryon densities, $n_{B}+n_{\bar{B}}$, of approximately $0.12 / \mathrm{fm}^{3}$ [26];

(ii) a self-consistent equation for the densities based on geometric estimates using percolation theory [27];

(iii) a fixed value of the entropy density, $s / T^{3}$, of approximately 7 [28-32].

All of these proposals have been used with considerable success, and it is the purpose of the present article to study and compare these different criteria for particle freeze-out to constrain their validity on the basis of available experimental data and corresponding statistical model analyses. It is not always straightforward to judge their quality, since small variations in parameters can sometimes lead to substantial shifts in results. In our analysis, we quantify the explicit dependence of different freeze-out criteria on excluded volume corrections, the resonance composition of the fireball, strangeness content and the corrections related to the canonical implementation of strangeness conservation. In particular, excluded volume corrections $[33,34]$ have a very strong impact on the validity of most of the criteria.

We show that in the energy range above the top AGS beam energy and within present accuracies, it is rather difficult to distinguish between different freeze-out conditions. However, the lower energy heavy-ion data favor the constant energy per particle as the unified condition of chemical particle freezeout. It also exhibits the least sensitivity to model assumptions and the most stability to small changes in the values of the parameters.

\section{GLOBAL DESCRIPTION}

The results from particle yields obtained by different groups for the thermal parameters are not always identical. The differences are small and never exceed the level of a few percent. Nevertheless, such differences play a role when one tries to determine unified descriptions over a wide range of beam energies. The origin of these differences has not been fully studied until now. However, to a large extent these differences could be attributed to different assumptions on the number of resonances, their widths, the treatment of weak decays, the description of strangeness saturation and how, or if at all, repulsive interactions, in the form of hard core, excluded volume corrections, have been included in the calculations. To pin down the differences would involve a very detailed knowledge of programming codes. This is not yet possible, as codes are not always made available. In order to have a consistent presentation, we have made some choices which are biased by our own experience: the code used in this paper is available for inspection [35]. Our analysis relies on the most recent results obtained in statistical-thermal model fits to $\mathrm{Au}+\mathrm{Au}$ and $\mathrm{Pb}+\mathrm{Pb}$ systems, performed by numerous groups over a wide range of energies [5-14,17]. These results are summarized in Table I and are also included in Fig. 1.

Before discussing regularities in Table I, we present first a polynomial fit using the checked entries in Table I as input. This gives

$$
T\left(\mu_{B}\right)=a-b \mu_{B}^{2}-c \mu_{B}^{4},
$$

where $a=0.166 \pm 0.002 \mathrm{GeV}, b=0.139 \pm 0.016 \mathrm{GeV}^{-1}$, and $c=0.053 \pm 0.021 \mathrm{GeV}^{-3}$. The $\sqrt{s}=200 A \mathrm{GeV}$ points were not included in the determination of Eq. (1). The comparison of the above equation with the $\sqrt{s}$-dependent chemical potential from Table I is shown in Fig. 1.

It is also straightforward to verify that at $\sqrt{s}=200 \mathrm{GeV}$ Eq. (1) is consistent within errors with the expected results from thermal model analyses. The energy dependence of the baryon chemical potential can be parametrized as [36]

$$
\mu_{B}(\sqrt{s})=\frac{d}{1+e \sqrt{s}}
$$

with $d=1.308 \pm 0.028 \mathrm{GeV}$ and $e=0.273 \pm 0.008 \mathrm{GeV}^{-1}$. The temperature $T$ and the baryon chemical potential $\mu_{B}$ are shown in Fig. 2 as a function of the beam energy.

It provides a good quantitative description of the corresponding thermal model results. Eqs. (1) and (2) lead to the energy dependence of the chemical freeze-out temperature and baryon chemical potential shown in Fig. 2. In heavy ion collisions at the LHC Eq. (2) leads to a value $\mu_{B} \approx 1 \mathrm{MeV}$. The parametrization given above should of course be used with caution, as small changes in the parameters lead to a significant change in the energy dependence, since particle yields depend on $\mu_{B}$ and $T$ exponentially.

Having established a basic parametrization of freeze-out conditions at different energies, we now proceed to compare various unified conditions for the chemical freeze-out curve. In the next section we will discuss how the freeze-out criteria mentioned in the introduction live up to a comparison with the description presented here. We will, in particular, discuss the dependence on parameters such as the strangeness suppression factor $\gamma_{s}$, canonical corrections to strangeness conservation, the cut-off of the hadronic mass spectrum and, finally, excluded volume effects. Clearly, a superficial examination 
TABLE I. Results obtained in statistical-thermal model fits to $\mathrm{Au}+\mathrm{Au}$ and $\mathrm{Pb}+\mathrm{Pb}$ collision systems by numerous groups over a wide range of energies. The checked entries have been included in the fits to determine the $T\left(\mu_{B}\right)$ and $\mu_{B}(\sqrt{s})$ parametrizations [Eqs. (1) and (2), respectively].

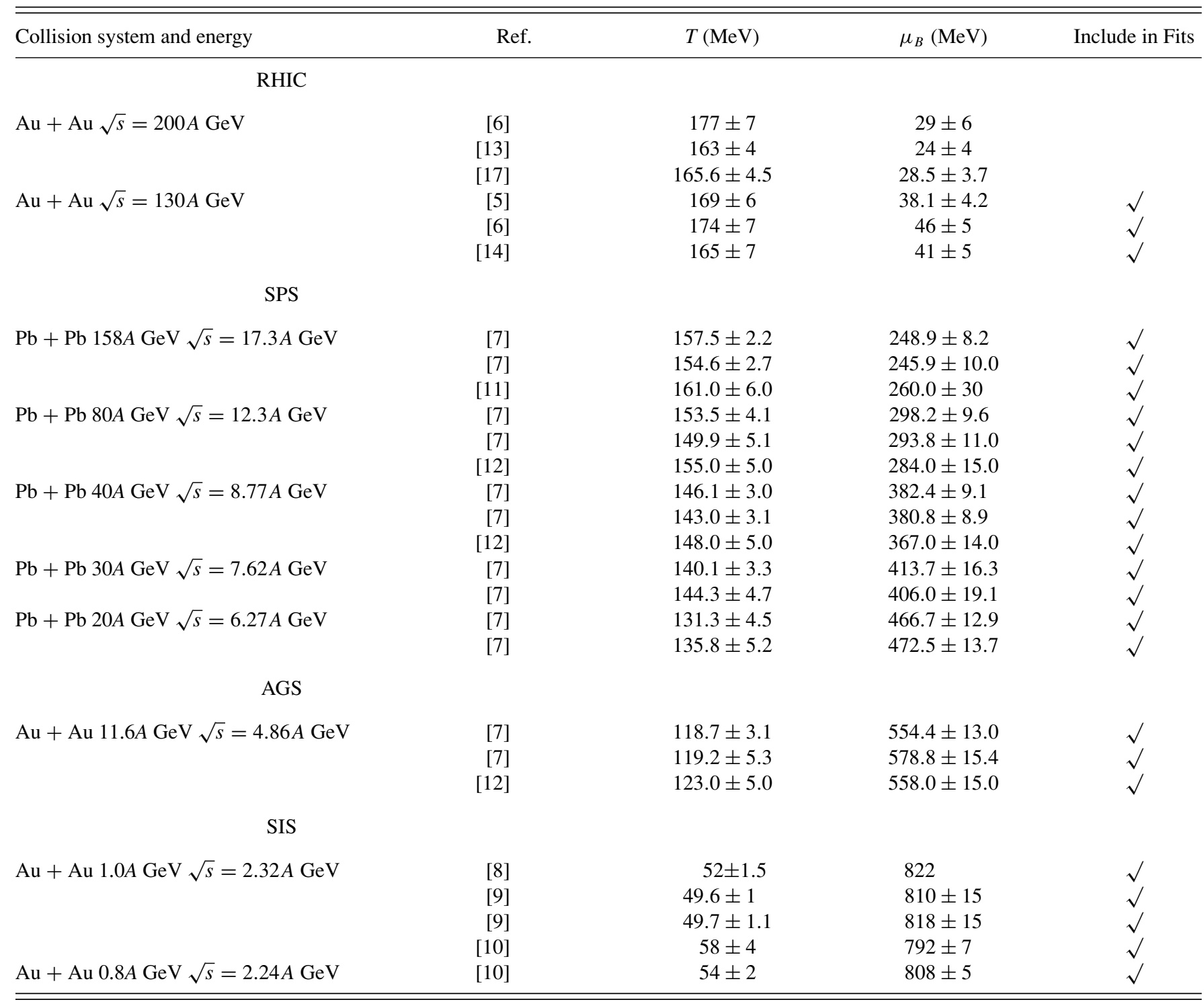

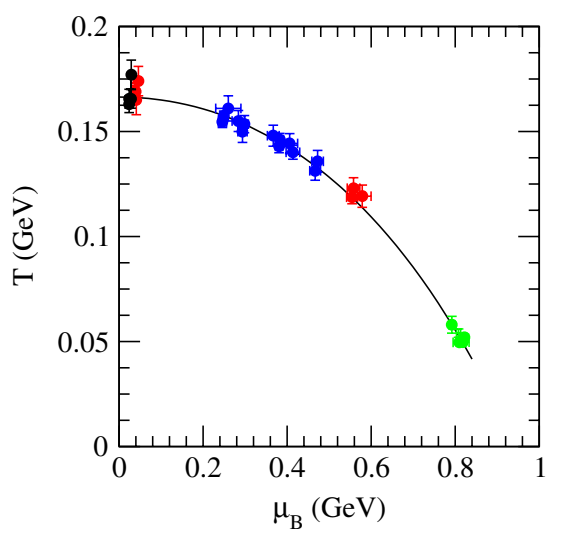

FIG. 1. (Color online) Values of $\mu_{B}$ and $T$ for different energies. shows that many criteria can be remarkably effective at describing chemical freeze-out in the restricted energy range. The largest discrepancies, as it will be argued, appear at low energies. However, a closer examination reveals that some criteria are more robust than others when small changes in parameters are allowed.

\section{DESCRIPTION OF FREEZE-OUT CRITERIA}

All criteria considered here are based on the hadron resonance gas model that was successfully used to describe particle yields and their ratios in heavy-ion collisions. Although this model is well known in the literature, for completeness we will summarize the basic ideas and formulas required to quantify each freeze-out condition. 


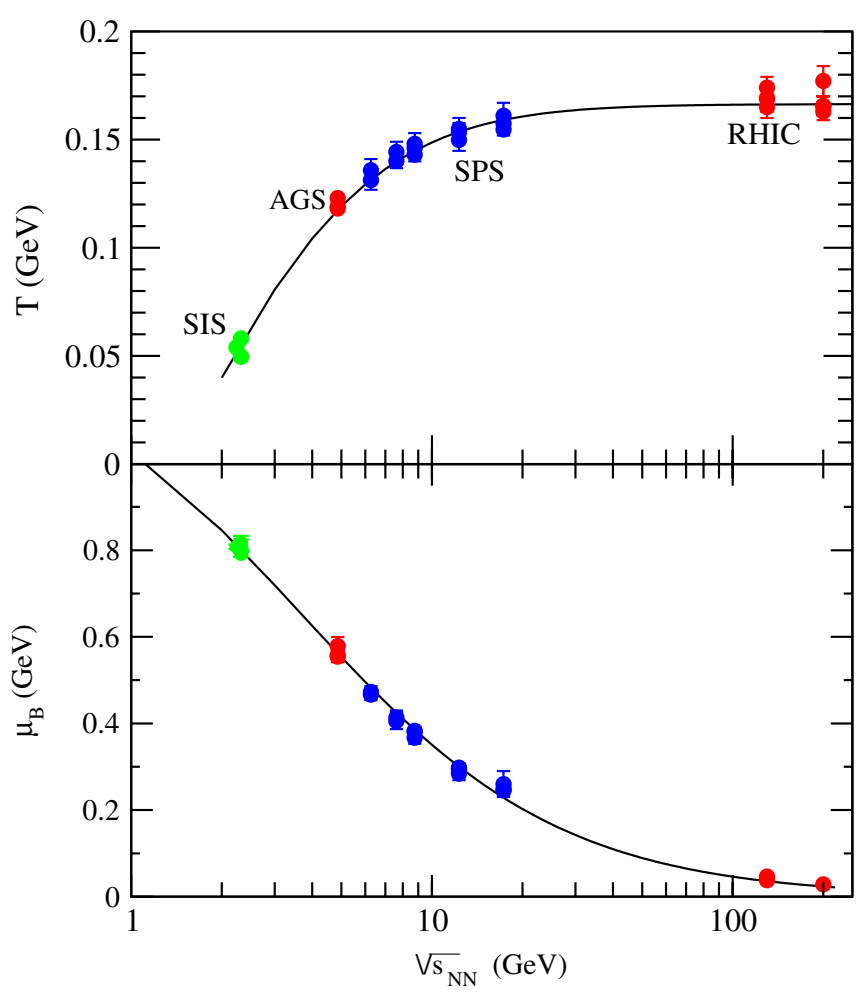

FIG. 2. (Color online) Energy dependence of the chemical freezeout parameters $T$ and $\mu_{B}$. The curves have been obtained using a parametrization discussed in the text.

The model is defined by the partition function of a resonance gas with free particle dispersion relations for all constituents [1]. The resulting observables, however, account for interactions between hadrons, to the extent that the thermodynamics of an interacting system of elementary hadrons can effectively be approximated by a mixture of ideal gases of stable particles and resonances. Thus, the thermodynamical observables are expressed as a sum over all mesonic and baryonic degrees of freedom, as well as their known resonances.

For our further discussion, the relevant quantities are: the total particle density $n$, total density of baryons $n_{B}+n_{\bar{B}}$, as well as energy $\epsilon$ and net baryon number $n_{B}-n_{\bar{B}}$ densities. All of these observables are functions of temperature $T$ and chemical potentials $\mu_{B}, \mu_{Q}$ and $\mu_{S}$ related to conservation of baryon number, electric charge and strangeness, respectively. The last two potentials are fixed from the initial conditions. Thus, we are left with only two independent variables: $T$ and $\mu_{B}$. The results for all relevant quantities obtained from the hadron resonance gas partition function are as follows:

The particle density is given by

$$
n=\sum_{i} g_{i} \int \frac{d^{3} p}{(2 \pi)^{3}} f_{i}
$$

the entropy density by

$s=\sum_{i} \frac{g_{i}}{2 \pi^{2}} \int_{0}^{\infty} p^{2} d p\left[-f_{i} \ln f_{i} \mp\left(1 \mp f_{i}\right) \ln \left(1 \mp f_{i}\right)\right]$, and, finally, the energy density,

$$
\epsilon=\sum_{i} g_{i} \int \frac{d^{3} p}{(2 \pi)^{3}} E_{i} f_{i}
$$

where

$$
f_{i}=\frac{1}{e^{\left(E_{i}-\mu_{i}\right) / T} \pm 1},
$$

with the upper (lower) signs being for Fermi-Dirac (BoseEinstein) statistics. The total density of baryons is calculated as in Eq. (3), but restricting the sum to baryons $n_{B}$ and their antiparticles $n_{\bar{B}}$. All of these results correspond to the grand-canonical formulation of the conservation laws. For central heavy-ion collisions at energies below AGS, strangeness conservation on average is not adequate and has to be implemented exactly in terms of the canonical ensemble [8]. A more general result for basic thermodynamic quantities in the canonical formulation of strangeness conservation can be found in Ref. [1]. The thermal particle phase-space of hadronic resonances has also to be modified by including finite width [1,37]. This modification amounts to convoluting the particle momentum distribution in Eqs. (3)-(5) with the Breit-Wigner resonance form-factor.

The strange particle phase-space in Eqs. (3)-(5) is controlled by the $\gamma_{s}$ parameter [38]. The value of $\gamma_{s}=1$ corresponds to a chemical equilibrium distribution, whereas $\gamma_{s} \neq 1$ indicates a deviation from chemical equilibrium. In addition, Eqs. (3)-(5) are only valid for point-like particles. Considering hadrons as extended objects would require an implementation of interactions. This can be done in the hard-core [34] and self-consistent implementation [33]. The first approach amounts to the multiplication of all results in Eqs. (3), (4), and (5) by a factor

$$
F\left(T, \mu_{B}, V_{0}\right)=\frac{1}{1+V_{0} n\left(T, \mu_{B}\right)},
$$

where the repulsive strength is determined by the volume parameter $V_{0}$.

In the self-consistent implementation of the repulsive interaction there is, in addition, a shift in chemical potential that guarantees that all observables Eqs. (3)-(5) are obtained as corresponding derivatives from the partition function [33]. The explicit results and a detailed description can be found in Ref. [33].

Before applying the above results to quantify different freeze-out criteria and comparing their predications with the thermal model analysis of heavy-ion data, we first briefly summarize basic concepts of these criteria.

\section{A. Percolation model}

Percolation theory was successfully applied to the description of critical properties of QCD matter [39]. In particular, color deconfinement in pure gauge theory can be treated as a percolation phenomenon [40]. The models based on percolation theory also provide a qualitative description of charmonium production and suppression in heavy-ion collisions [41]. 
In Ref. [27], percolation theory was used to formulate and quantify chemical freeze-out conditions in heavy-ion collisions. It is assumed that, in the sector of vanishing baryon density, hadronic matter freezes out according to the resonance gas approximation and by vacuum percolation, while the region of finite baryon density freezes out according to baryon percolation. The condition that describes the freeze-out line in heavy-ion collisions has been formulated as [27]

$$
n(T, \mu)=\frac{1.24}{V_{h}}\left[1-\frac{n_{B}(T, \mu)}{n(T, \mu)}\right]+\frac{0.34}{V_{h}}\left[\frac{n_{B}(T, \mu)}{n(T, \mu)}\right] .
$$

The volume $V_{h}$ is the hadronic size, which corresponds to a radius of approximately $r_{h} \approx 0.8 \mathrm{fm}$. The numbers 1.24 and 0.34 appearing in Eq. (8) are calculated within percolation theory [47]. The smallest one is determined when the size of the largest cluster falls below the size of the overall spatial volume. On the other hand, the number 1.24 is determined by the disappearance of any large-scale vacuum, and only the strongly interacting medium spans the entire space.

Equation (8), together with the results of the statisticalthermal model of the hadron resonance gas on the total density of all hadrons $n\left(T, \mu_{B}\right)$ and baryon number density $n_{B}\left(T, \mu_{B}\right)$, provide a unique solution $T=T\left(\mu_{B}\right)$ that characterizes the chemical freeze-out line in the $\left(T, \mu_{B}\right)$-plane within the percolation model.

\section{B. Fixed baryon+Antibaryon density}

The contributions of baryons to thermodynamics is usually characterized by the net baryon density, i.e., the difference between the density of baryons $n_{B}$ and antibaryons $n_{\bar{B}}$. In heavy-ion collisions, this varies strongly with collision energy. At SIS it is $\sim 1 / 3$ of nuclear matter density $\left(\rho_{0}=0.17 / \mathrm{fm}^{3}\right)$ and it strongly increases to reach a maximum at top AGS energies. It then gently decreases up to RHIC, where it is only $\sim 1 / 10$ of $\rho_{0}$. Due to baryon number conservation $\left(B=A_{\text {part }}\right)$, the variation of $n_{B}-n_{\bar{B}}=B / V$ reflects the corresponding change in the volume of the fireball. Indeed, a comparison of the total yields of different particle species in heavy-ion collisions with calculations in the statistical model, or, alternatively, using two pion correlations, has shown that the volume has a minimum between AGS and the lowest SPS energy [42] and exhibits a dependence on $\sqrt{s}$ opposite to that of $n_{B}$.

Despite this large variation, it was first noticed in Ref. [26] that the sum of baryon and antibaryon densities, $n_{B}+n_{\bar{B}}$, remains remarkably constant. Thus, it was proposed [26] that in heavy-ion collisions the chemical freeze-out curve can be obtained from the condition,

$$
n_{B}+n_{\bar{B}} \simeq 0.12 \mathrm{fm}^{-3},
$$

of fixed density of total number of baryons and antibaryons.

The observation [42] that Eq. (9) is satisfied in high energy heavy-ion collisions was important phenomenologically to explain the equivalence at 160 and $40 \mathrm{~A} \mathrm{GeV}$ of the enhancement of the low mass dilepton yield observed by the CERES Collaboration in Au-Pb collisions [44] and recently by NA60 in In-In reactions [45]. Theoretically, the enhancement was argued [46] to be due to rho meson broadening through its rescattering with surrounding baryons. Thus, the broadening is determined by the total density of baryons. The fact that in $\mathrm{Au}-\mathrm{Pb}$ collisions at 160 and $40 \mathrm{~A} \mathrm{GeV}$ the total baryon density is comparable, gave an argument for the observed similar structure of the low mass dilepton yield at these energies.

Clearly, in low energy heavy-ion collisions between SIS and AGS, the production of antibaryons is suppressed. Thus, in this energy range the net baryon number density, to a good approximation, is equivalent to the total density of baryons, that is $n_{B}+n_{\bar{B}}$. Consequently, since the volume at freeze-out from SIS to AGS extracted from HBT correlations [42] drops by a factor $1 / 3$, there should be a corresponding increase in $n_{B}+n_{\bar{B}}$. Because of the above, the freeze-out condition (9) is not expected to work in low energy heavy-ion collisions see, e.g., figure 20 in Ref. [43]. The question remains as to at what energy the violation of Eq. (9) as the condition of particle freeze-out sets in. The answer to this question requires a quantitative analysis, which is given in the next section.

\section{Fixed entropy density over $T^{3}$}

The chemical freeze-out condition of fixed entropy density over temperature to the third power,

$$
\frac{s}{T^{3}} \simeq 7
$$

was proposed independently from two very different considerations. In Refs. [31,32] it was used to extrapolate lattice gauge results from $\mu_{B}=0$ to finite values of $\mu_{B}$ by keeping $s / T^{3}$ fixed. In Refs. [28,29] the entropy density was used to separate between a baryon-dominant region and a meson-dominant one, in order to understand the rapid change in certain particle ratios observed at lower SPS energies by the NA49 Collaboration [48]. In the course of this analysis, it was noted that, over a broad energy range, the total entropy density divided by $T^{3}$ remained constant, despite very large changes in the baryon chemical potential and the temperature.

The freeze-out condition (10) is rather surprising. In the ideal gas of massless constituents and for a net baryon free system, the $s / T^{3}$ ratio describes the number of degrees of freedom. In the case of massive particles and at finite chemical potential, this ratio can be parameterized as $s / T^{3} \simeq a\left(T, \mu_{B}\right)$. Thus, for fixed $T$ and $\mu_{B}$, the parameter $a\left(T, \mu_{B}\right)$ describes the effective number of degrees of freedom in a system. The condition (10) tells us that this effective number of degrees of freedom $a\left(T, \mu_{B}\right)$ is common for all collision energies. In the thermodynamical context, this also means that $a\left(T, \mu_{B}\right)$ is independent of thermal parameters at chemical freeze-out. This is quite unexpected, as changing the collision energy implies not only changing the thermal parameters but also the hadronic composition of the fireball. Naively one would expect that in such a case the number of degrees of freedom $a\left(T, \mu_{B}\right)$ should be a strongly varying function of collision energy.

\section{Fixed $\langle E\rangle /\langle N\rangle \simeq 1 \mathrm{GeV}$}

This criterion was first proposed in Refs. [24,25] as a link unifying results obtained at SIS with top AGS and SPS. Since 
then, the description has proven its validity also at RHIC $[5,6]$ and at the lower SPS energies $[1,7,49]$. This condition was successfully used in the literature to make predictions [49] of freeze-out parameters at SPS energies of 40 and $80 \mathrm{~A} \mathrm{GeV}$ for $\mathrm{Pb}-\mathrm{Pb}$ collisions long before the data were taken. These predictions turned out to be in agreement with the statistical model analysis of recent experimental data obtained at these collision energies [7].

The reason why $\langle E\rangle /\langle N\rangle$ is constant in heavy-ion collisions from SIS up to RHIC can be intuitively explained as follows: In a nonrelativistic system $\langle E\rangle /\langle N\rangle \simeq\langle m\rangle+\frac{3}{2} T$, with $\langle m\rangle$ being the average mass in the thermal fireball [50]. At SIS, the thermal mass is of the order of the nucleon mass, whereas the freeze-out temperature $T_{f} \simeq 50 \mathrm{MeV}$. Thus, at SIS the $\langle E\rangle /\langle N\rangle$ ratio is indeed comparable with $1 \mathrm{GeV}$. At SPS and RHIC, the leading particles in the final state are pions. However, at chemical freeze-out most of the pions are still hidden in the mesonic and baryonic resonances. Thus, here the average thermal mass corresponds approximately to the $\rho$-meson mass. Consequently, with $T_{f} \sim 160-170 \mathrm{MeV}$ a comparable value of $\langle E\rangle /\langle N\rangle$ is obtained at SPS and RHIC as in a much lower energy at SIS.

We have already indicated that the chemical freeze-out line dynamically coincides with the inelasticity line in heavyion collisions. Thus, also the $\langle E\rangle /\langle N\rangle \simeq 1 \mathrm{GeV}$ condition should have the above dynamical interpretation. Recently, the correlation between the $\langle E\rangle /\langle N\rangle \simeq 1 \mathrm{GeV}$ condition and inelasticity has been investigated in central $\mathrm{Pb}-\mathrm{Pb}$ collisions at the SPS in terms of the ultrarelativistic quantum molecular dynamics model (UrQMD) [51]. A detailed study has shown that there is a clear correlation between the chemical break-up in terms of inelastic scattering rates and the rapid decrease in energy per particle. If $\langle E\rangle /\langle N\rangle$ approaches the value of $1 \mathrm{GeV}$ the inelastic scattering rates drop substantially and further evolution is due to elastic collisions that preserve the chemical composition of the collision fireball. Following the above UrQMD results, it is conceivable that $\langle E\rangle /\langle N\rangle \simeq$ $1 \mathrm{GeV}$ is related with inelasticity in heavy-ion collisions. This could dynamically justify the applicability of this freeze-out criterion.

It was noted recently in Ref. [12] that the results from RHIC favor a higher value for $\langle E\rangle /\langle N\rangle$. This was interpreted as a change in the baryonic composition of a two-component thermal source. We find that a consistent description using $\langle E\rangle /\langle N\rangle=1.08 \mathrm{GeV}$ is quite adequate and there is no need for different values at different energies. The comparison of each of these freeze-out criteria with all known freeze-out parameters is shown in Fig. 3.

\section{SENSITIVITY AND ROBUSTNESS OF FREEZE-OUT CRITERIA}

The chemical freeze-out criteria discussed in the last section are not quite model independent. Their predictions are sensitive to various assumptions and to the choice of parameters. In this section, we present a detailed discussion of the robustness of these criteria. In this context, we consider the influence of excluded volume corrections, strangeness suppression and

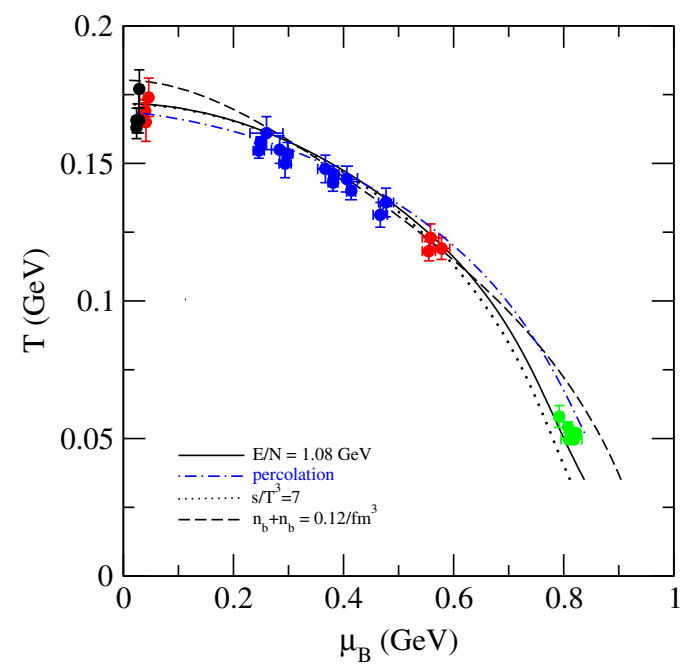

FIG. 3. (Color online) Description of chemical freeze-out by four different criteria discussed in the text.

hadronic mass spectrum cut-off on the predictions of the different freeze-out criteria.

\section{A. Sensitivity on excluded volume corrections}

The $\langle E\rangle /\langle N\rangle$ criterion involves the ratio of two extensive quantities and, hence, is nearly independent of excluded volume corrections. In the Boltzmann approximation, these corrections cancel exactly, provided that the same proper volume parameter is applied. Otherwise, for example, pions and protons pick up different correction factors through the shift in chemical potentials. In quantum statistics, the shift in chemical potentials always leads to a (small) change. The energy per particle is also not strongly influenced if repulsive interactions are implemented through a mean-field potential.

The freeze-out condition of fixed entropy density divided by $T^{3}$, as well as that of fixed total density of baryons plus antibaryons, use extensive thermodynamic variables. Hence, the dependence on the excluded volume corrections is, in general, not negligible. However, as it turns out, only a moderate dependence is found for the constant $n_{B}+n_{\bar{B}}$ criterion, which is shown in Fig. 4. The repulsive strength of hadrons is parametrized there by the radius parameter. The freeze-out curve moves to higher values of $T$ as the radius increases. A change in radius of $0.1 \mathrm{fm}$ easily leads to a change in temperature which can be larger than $10 \mathrm{MeV}$. The repulsive interactions suppress thermal particle phase-space. Thus, to reproduce the same value of total density of baryons, one needs to increase temperature at fixed $\mu_{B}$.

The percolation model criterion is determined by the total particle and the net baryon number density. It thus shows a similar dependence on excluded volume radius as discussed above. This model is based on geometric considerations which are affected by volume parameters. The changes are almost identical to the previous case and are not shown explicitly.

Contrary to the percolation and fixed baryon density conditions, the constant $s / T^{3}$ criterion shows a wild dependence on the excluded volume radius (as seen in Fig. 4). This casts 


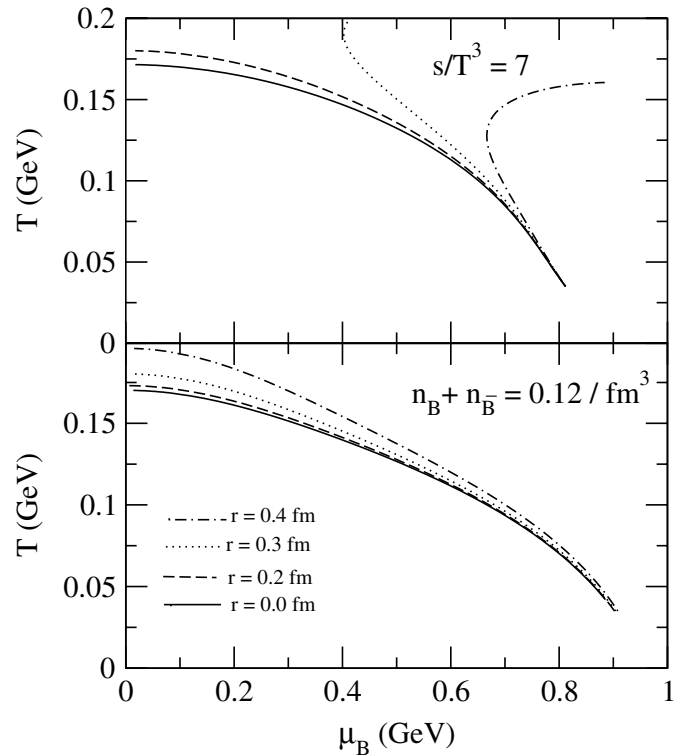

FIG. 4. The effect of excluded volume corrections on the constant $n_{B}+n_{\bar{B}}$ (bottom) and constant $s / T^{3}$ (top) freeze-out criterion.

considerable doubt on the value of this criterion for model building. It is clear that only the complete neglect of excluded volume corrections leads to a realistic freeze-out curve. It is well known, however, that such corrections cannot be ignored, especially at low temperatures, as this leads, e.g., to a totally unrealistic picture for the phase transition [52] and is also inconsistent with the properties of the nuclear potential.

\section{B. Sensitivity on resonance spectrum mass cut-off}

The thermal-statistical model relies very strongly on input from the particle data table [53]. Heavy resonances are, in general, suppressed by the Boltzmann factor $e^{-E / T}$. However, when the temperature or baryon chemical potential increase, the contribution of heavy resonances also increases and this becomes especially noteworthy in the small $\mu_{B}-$ high $T$ region. As the information about heavy resonances is fragmentary, several analyses have introduced a cut-off in masses, such that all resonances with mass heavier than this cut-off mass are not taken into account in the thermal-statistical model. In particular, if all resonances listed in the particle data booklet are taken into consideration, we refer to this as the "No Mass Cut" model. The effects of this mass cut-off are similar for all criteria considered. As an example, we show the influence on the $\langle E\rangle /\langle N\rangle=1 \mathrm{GeV}$ criterion and on the finite density $n_{B}+n_{\bar{B}}=0.12 / \mathrm{fm}^{3}$ criterion in Fig. 5 . It is seen in Fig. 5 that the largest influence of the mass cut in the hadron spectrum appears at high temperature and low $\mu_{B}$. At fixed $\mu_{B}$, the changes in the freeze-out temperature never exceed $10 \mathrm{MeV}$.

The cut-off on the hadronic mass spectrum in the thermalstatistical model is particularly relevant when calculating particle yields. This is because the decay properties of heavier resonances into lighter hadrons are needed and are not always well established. However, for the description of basic

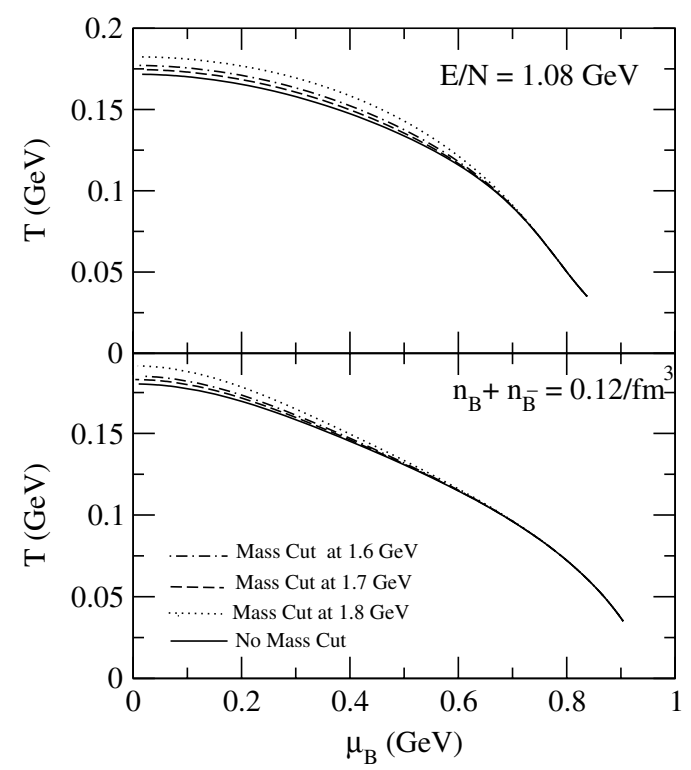

FIG. 5. The effect of the resonance spectrum mass cut-off on the chemical freeze-out curve: top constant $\langle E\rangle /\langle N\rangle$, bottom $n_{B}+n_{\bar{B}}$ freeze-out criterion.

thermodynamic observables characterizing the properties of the hadronic medium, like the energy, entropy, particle and baryon number density, the only resonance characteristics needed are the masses, degeneracy factors and quantum numbers. In most cases, this information is known even for heavy mesonic and baryonic states.

\section{Sensitivity on strangeness suppression}

Strange particle yields, their ratios and momentum distributions are relevant observables that characterize the origin, composition and production dynamics in heavy-ion collisions $[1,38]$. However, their contribution to global thermodynamic observables, summarized in Eqs. (3)-(5), is not essential. In low energy heavy-ion collisions at SIS up to AGS, strange particles are only rarely produced and can be almost completely neglected when considering global thermodynamic characteristics of the collision fireball. At SPS and RHIC, due to the much higher temperature, the strangeness degrees of freedom contribute about $25 \%$ to the total particle density. Due to this, one should not expect very large changes in the predictions of different freeze-out criteria when modifying the strange particle content.

In the statistical model under consideration, a modification of the strange particles from chemical equilibrium is described by the $\gamma_{s}$ factor or by the canonical constraints imposed by exact strangeness conservation.

The strangeness-suppression (or enhancement) factor $\gamma_{s}$ modifies the multiplicities of strange and anti-strange hadrons, parameterizing a deviation from chemical equilibrium, in the Boltzmann approximation, in the manner given by the equation below:

$$
\left\langle n_{i}\right\rangle=\gamma_{s}^{n_{s}}\left\langle n_{i}\right\rangle_{\text {equilibrium }} .
$$




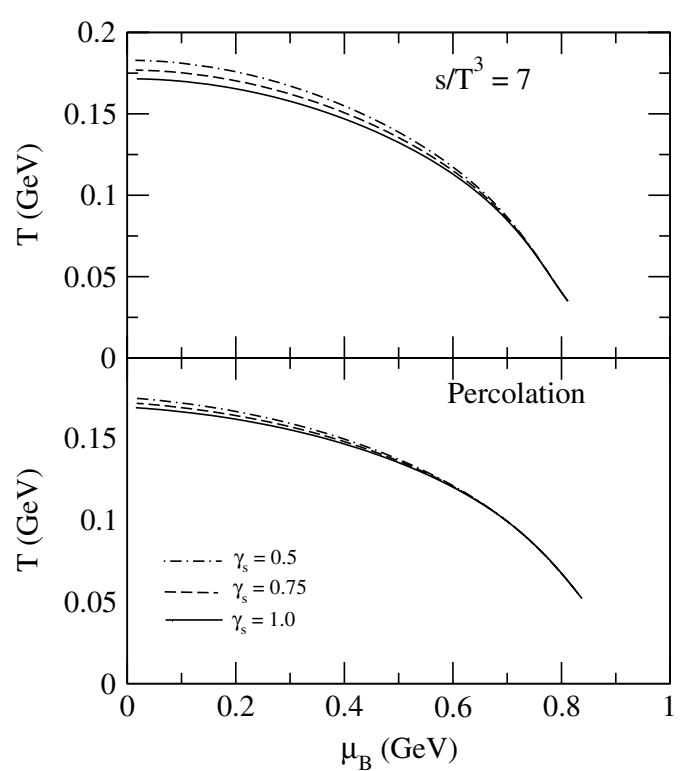

FIG. 6. The effect of varying $\gamma_{s}$ on the constant $s / T^{3}$ (top) and the percolation criteria (bottom).

It does not affect the nonstrange resonances since $n_{s}=0$ in this case. Its influence on the chemical freeze-out curve is therefore small and, at low energies, negligible. This is shown for the $s / T^{3}$ criterion and for the percolation model in Fig. 6 . The effect on the other freeze-out criteria is similar and not shown explicitly.

For small systems, various corrections become important. In our considerations, these affect mainly the production of strange particles in heavy-ion collisions at low energies due to the very small number of produced particles. In this case, strangeness conservation should be treated exactly, which results in so-called canonical corrections. These depend on the volume over which strangeness is balanced. This correlation volume does not need to be as large as the volume of the overall system. Several calculations have been made where strangeness conservation is imposed over volumes which are smaller than the overall volume. These are parametrized by a canonical radius. In Fig. 7, one typical example shows the influence of this canonical radius on the freeze-out criteria.

Similarly as for the strangeness suppression factor, since the relative number of strange particles is small, the effect of the canonical radius on the chemical freeze-out curve is small. All criteria are only weakly dependent on variations in the canonical parameter. Small differences are only seen above AGS energies, where the contribution of the strangeness sector is not negligible. At low energy, this effect is entirely irrelevant. However, for the discussion of strange particles or their yields, the influence of canonical effects is essential $[8,11,49,54]$. This is particularly the case for multistrange baryons in low energy central collisions, as well as in peripheral $A-A$ collisions at higher energies $[49,54]$. The $\gamma_{s}$ factor has a similar influence on strange and multistrange particle yields [3,7].

Comparing different freeze-out criteria, we have been bound by the knowledge of the lowest chemical freeze-out parameters at SIS energy. Clearly, the thermal-statistical model

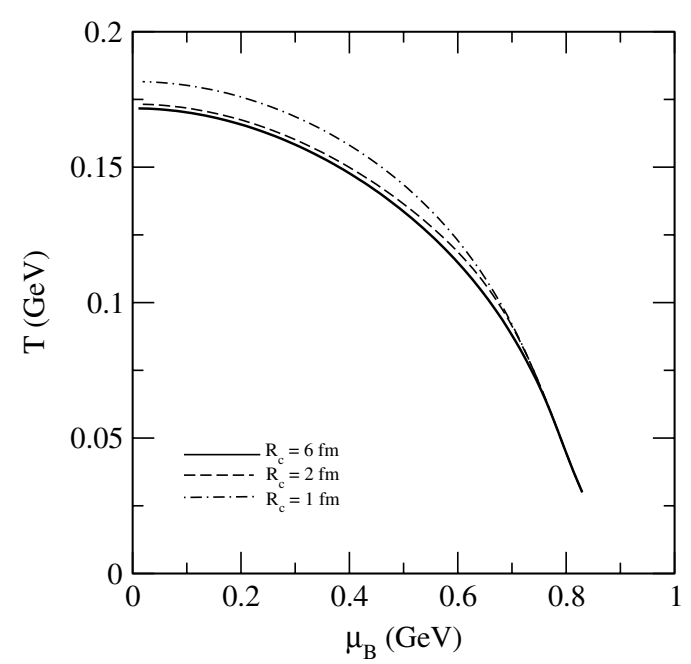

FIG. 7. The effect of the canonical radius on the constant $s / T^{3}$ freeze-out criterion.

considered here is limited and cannot be used if the assumption of energy conservation on the average is not valid. This indeed is the case in low energy heavy-ion collisions, where the full microcanonical formulation [55] of the partition function is required. This also means that the extrapolation of different freeze-out criteria to the $T \simeq 0$ limit and within the grandcanonical formulation is questionable. Until now the model has shown its validity in the description of heavy-ion data up to beam energies of about $800 \mathrm{MeV}$.

There is a class of further criteria (e.g., the constant energy or total particle number density) that are consistent with the freeze-out parameters in the energy range above the lowest SPS energies. However, since these conditions are based on intensive observables, they suffer from variations with the parametrization of repulsive interactions. In addition, both of these criteria are inconsistent with the SIS freeze-out parameters.

\section{SUMMARY AND CONCLUSIONS}

From the results presented above one can conclude that, on a phenomenological level, the various freeze-out criteria give quite satisfactory descriptions of the particle multiplicities measured in heavy-ion collisions. Deviations can be reduced by suitably modifying input parameters in the different models. However, it is clear that some of the criteria are not very stable or robust when one applies small changes to the input considerations and are, therefore, more model dependent.

A further method to differentiate freeze-out conditions would be to calculate particle excitation functions. The differences between such criteria would be even more transparent, as some particle excitation functions are very sensitive to the value of the thermal parameters. A good example is the $K / \pi$ ratio which at SIS energy is a very sensitive probe of temperature and canonical suppression effects. The energy per particle criterion has been shown to provide a consistent description of the particle excitation functions over the whole energy range. Some of the results of this criterion, like the 
energy dependence of the $\Lambda / \pi$ and $K^{+} / K^{-}$ratios for different centrality and collision energies, have been confirmed by recent heavy-ion data.

The main differences between the chemical freeze-out criteria analyzed here appear in the region of low temperature and large baryon chemical potential, that is for SIS up to AGS energy. This region obviously favors the $\langle E\rangle /\langle N\rangle$ criterion as the most appropriate one to describe chemical freeze-out over all energies. This is supported quantitatively by the fact that this freeze-out condition has the lowest $\chi^{2}$ per degree of freedom of all the criteria considered in this analysis and was shown to provide a good description of different particle excitation functions in heavy-ion collisions. The energy per particle is also consistent through transport model calculations with the dynamical interpretation of chemical freeze-out as the inelasticity condition in heavy-ion collisions.

\section{ACKNOWLEDGMENTS}

JC acknowledges the financial support of the Alexander von Humboldt Foundation and the Italy-South Africa co-operation programme and discussions with F. Becattini. KR also acknowledges stimulating discussions with P. Braun-Munzinger, H. Satz and J. Stachel. Two of us (JC and SW) would like to thank the theory division of the GSI for their hospitality. The partial support of the Polish Committee for Scientific Research under contract 2P03 (06925) and the Polish-South-African Research Project is acknowledged.
[1] P. Braun-Munzinger, K. Redlich, J. Stachel, in Quark Gluon Plasma 3, edited by R. C. Hwa and X. N. Wang (World Scientific Publishing, Singapore, 2004), nucl-th/0304013.

[2] J. Cleymans and H. Satz, Z. Phys. C 57135 (1993).

[3] F. Becattini, J. Cleymans, A. Keranen, E. Suhonen, and K. Redlich, Phys. Rev. C 64, 024901 (2001).

[4] P. Braun-Munzinger, J. Stachel, J. P. Wessels, and N. Xu, Phys. Lett. B344, 43 (1995); B365, 1 (1996); P. Braun-Munzinger, I. Heppe, and J. Stachel, ibid. B465, 15 (1999).

[5] J. Cleymans, B. Kämpfer, M. Kaneta, S. Wheaton, and N. Xu, Phys. Rev. C 71, 054901 (2005).

[6] P. Braun-Munzinger, D. Magestro, K. Redlich, and J. Stachel, Phys. Lett. B518, 41 (2002).

[7] F. Becattini, M. Gaździcki, A. Keränen, J. Manninen, and R. Stock, Phys. Rev. C 69, 024905 (2004); J. Manninen, F. Becattini, and M. Gaździcki, hep-ph/0511092.

[8] J. Cleymans, H. Oeschler, and K. Redlich, Phys. Rev. C 59, 1663 (1999); K. Redlich and L. Turko, Z. Phys. C 5, 201 (1980).

[9] F. Becattini, J. Cleymans, A. Keränen, E. Suhonen, and K. Redlich, Phys. Rev. C 64, 024901 (2001).

[10] R. Averbeck, R. Holzmann, V. Metag, and R. S. Simon, Phys. Rev. C 67, 024903 (2003).

[11] I. Kraus, contribution to HEP2005, Europhysics Conference, July 21-27, 2005, Lisboa, Portugal, to be published in the proceedings of the conference.

[12] L. Bravina, A. Faessler, C. Fuchs, E. Zabrodin, and Z. D. Lu, Phys. Rev. C 66, 014906 (2002).

[13] J. Adams et al. (STAR Collaboration), Nucl. Phys. A 757, 102 (2005)

[14] W. Broniowski, W Florkowski, and M. Michalec, Acta Phys. Pol. B 33, 761 (2002).

[15] A. Dumitru, L. Portugal, and D. Zschiesche, nucl-th/0511084.

[16] L. V. Bravina, E. E. Zabrodin, S. A. Bass, M. Bleicher, M. Brandstetter, S. Soff, H. Stocker, and W. Greiner, Phys. Rev. C 62064906 (2000).

[17] A. Baran, W. Broniowski, and W. Florkowski, Acta Phys. Pol. B 35, 779 (2004).

[18] C. R. Allton, M. Doring, S. Ejiri, S. J. Hands, O. Kaczmarek, F. Karsch, E. Laermann, and K. Redlich, Phys. Rev. D 71, 054508 (2005); S. Ejiri, F. Karsch, and K. Redlich, hep-ph/0509051; K. Redlich, J. Phys. G 31, S943 (2005).
[19] F. Karsch, A. Tawfik, and K. Redlich, Phys. Lett. B571, 67 (2003); Eur. Phys. J. C 29, 549 (2003).

[20] J. Stachel, Nucl. Phys. A654, 119c (1999); P. Braun-Munzinger J. Stachel, J. Phys. G 28, 1971 (2002).

[21] K. Redlich, J. Phys. Conf. Ser. 5, 162 (2005); Nucl. Phys. A749, 14 (2005).

[22] P. Braun-Munzinger, J. Cleymans, H. Oeschler, and K. Redlich, Nucl. Phys. A697, 902 (2002).

[23] U. Heinz, Nucl. Phys. A685, 414 (2001); A661, 349 (1999).

[24] J. Cleymans and K. Redlich, Phys. Rev. Lett. 81, 5284 (1998).

[25] J. Cleymans and K. Redlich, Phys. Rev. C 60, 054908 (1999).

[26] P. Braun-Munzinger and J. Stachel, J. Phys. G: Nucl. Part. Phys. 28, 1971 (2002).

[27] V. Magas and H. Satz, Eur. Phys. J. C 32, 115 (2003).

[28] M. Stankiewicz, B. Sc. Honours Thesis, University of Cape Town (unpublished) 2004, nucl-th/0509058.

[29] J. Cleymans, H. Oeschler, K. Redlich, and S. Wheaton, Phys. Lett. B615, 50 (2005).

[30] J. Cleymans, M. Stankiewicz, P. Steinberg, and S. Wheaton, nucl-th/0506027.

[31] A. Tawfik, J. Phys. G: Nucl. Part. Phys. 31, S1105 (2005).

[32] A. Tawfik, hep-ph/0507252; hep-ph/050824.

[33] D. H. Rischke, M. I. Gorenstein, H. Stoecker, and W. Greiner, Z. Phys. C 51, 485 (1991).

[34] J. Cleymans, K. Redlich, H. Satz, and E. Suhonen, Z. Phys. C 33, 151 (1986).

[35] S. Wheaton and J. Cleymans, hep-ph/0407174; J. Phys. G: Nucl. Part. Phys. 31, S1069 (2005).

[36] S. Wheaton, The Development and Application of THERMUS - a Statistical-Thermal Model Analysis Package for ROOT, unpublished, University of Cape Town, August 2005.

[37] J. Cleymans, D. Elliott, A. Keranen, and E. Suhonen, Phys. Rev. C 57, 3319 (1998).

[38] J. Rafelski, Phys. Lett. B262 333 (1991); J. Rafelski, J. Letessier, and A. Tounsi, Acta Phys. Pol. B 27, 1037 (1996); P. Koch, B. Müller, and J. Rafelski, Phys. Rep. 142, 167 (1986).

[39] H. Satz, Nucl. Phys. A642, 130 (1998); H. Satz, Comput. Phys. Commun. 147, 46 (2002) and hep-ph/0212046. 
[40] S. Fortunato and H. Satz, Phys. Lett. B475, 311 (2000); Nucl. Phys. A681, 466 (2001).

[41] S. Digal, S. Fortunato, P. Petreczky, and H. Satz, Phys. Lett. B549, 101 (2002); S. Digal, S. Fortunato, and H. Satz, Eur. Phys. J. C 32, 547 (2004).

[42] A. Andronic and P. Braun-Munzinger, Lect. Notes Phys. 652, 35 (2004); D. Adamova et al. (CERES Collaboration), Phys. Rev. Lett. 90, 022301 (1003).

[43] A. Andronic, P. Braun-Munzinger, and J. Stachel, nuclth/0511071.

[44] G. Agakishiev et al. (CERES Collaboration), Eur. Phys. J. C 41, 475 (2005); D. Adamova et al., Nucl. Phys. A749, 160 (2005).

[45] S. Damjanovic (NA60 Collaboration), talk presented at Quark Matter 2005, Budapest, nucl-ex/0510044.
[46] R. Rapp, J. Phys. G 31, S217 (2005).

[47] M. Isichenko, Rev. Mod. Phys. 64, 961 (1992).

[48] M. Gazdzicki (NA49 Collaboration), J. Phys. G: Nucl. Part. Phys. 30, 701 (2004).

[49] K. Redlich and A. Tounsi, Eur. Phys. J. C 24, 589 (2002).

[50] R. Hagedorn, Thermodynamics of strong interactions, CERN Report 71-12 (1971).

[51] M. Bleicher and J. Aichelin, Phys. Lett. B530, 81 (2002).

[52] J. Cleymans, K. Redlich, H. Satz, and E. Suhonen, Z. Phys. C 58, 347 (1993).

[53] Particle Data Group, Phys. Lett. B592, 1 (2004).

[54] A. Tounsi and K. Redlich, Eur. Phys. J. C 24, 529 (2002); J. Phys. G 28, 2095 (2002).

[55] F. Becattini and L. Ferroni, Eur. Phys. J. C 38, 225 (2004). 